\title{
Langues et dialectes dans l'arc alpin
}

Tullio Telmon et Jeanine Médélice

\section{(2) OpenEdition}

Journals

Édition électronique

URL : http://journals.openedition.org/esp/2775

DOI : 10.4000/esp.2775

ISSN : 2532-0319

\section{Éditeur}

Centre d'Information sur l'Éducation Bilingue et Plurilingue

\section{Édition imprimée}

Date de publication : 1 décembre 2013

Pagination : 69-80

ISSN : 1127-266X

\section{Référence électronique}

Tullio Telmon et Jeanine Médélice, «Lanques et dialectes dans l'arc alpin », Éducation et sociétés plurilingues [En ligne], 35 | 2013, mis en ligne le 01 février 2020, consulté le 17 février 2021. URL http://journals.openedition.org/esp/2775 ; DOI : https://doi.org/10.4000/esp.2775 


\section{LANGUES ET DIALECTES DANS L'ARC ALPIN (1)}

Tullio Telmon, Jeanine MÉDÉLICE

Dopo la legge sulla tutela delle minoranze linguistiche storiche, approvata dal Parlamento italiano nel dicembre del 1999, tutte le lingue dell'uso popolare presentate in questo articolo, con la sola eccezione delle parlate galloitaliche e italo romanze, hanno assunto una forma di ufficialità. Esse possono infatti essere usate, per esempio, nei consigh comunali e neghi uffici municipali. Il vero problema è però quello delle varietà: se lo scopo della legge è quello di salvaguardare delle minoranze, lipotesi di privilegiare una varietà a scapito delle altre (o di trovare altre "soluzioni") appare assai poco coerente con $i$ suoi obbiettivi.

Since the Italian State law for the preservation of historical linguistic minorities, all the vernacular languages presented in this article (except those of the gallo-italic group and the italo-roman ones) have become official in a way, since they can be used for example in board meetings or town halls. The problem of varieties remains: if the aim of the law is to save minorities, the idea of giving precedence to one form above the others, or of finding other «solutionss, does not seem very coherent reith its objective.

LES LANGUES

DE L'USAGE

POPULAIRE

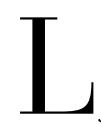

es manuels donnent souvent une image très simplifiée de la situation linguistique des Alpes; cette image ne tient presque jamais compte des entrecroisements entre langues de l'usage populaire, langues officielles, langues de la culture, langues écrites et langues exclusivement ou principalement orales. En principe, dans la plupart des cas, la caractéristique la plus particulière de la situation linguistique, soit sur le versant extérieur (français, suisse, austro-allemand, slovène) de la chaîne alpine, soit surtout sur l'intérieur (italien) est celle d'un plurilinguisme très répandu, qui dans la plupart des cas revêt l'aspect de diglossie. Les conditions les plus fréquentes sont celles de communautés linguistiques qui possèdent une langue locale à elles, et qui utilisent cette langue locale pour la communication à l'intérieur de leur village ou de leur famille, à côté d'au moins une deuxième langue, celle-ci apprise surtout par l'intermédiaire de l'enseignement scolaire, et utilisée pour les rapports avec l'administration et la culture.

Par cette définition un peu sommaire, nous voulons ici parler de la réalité linguistique qui, généralement, est comprise dans l'univers des dialectes. Quels que soient la famille, le groupe ou le sous-groupe linguistiques d'appartenance, chacun des dialectes locaux dont nous parle- 
rons devra être considéré comme une langue à part entière, puisqu’il remplit toutes les fonctions que remplit tout système linguistique, et puisqu'il possède, comme tout système linguistique, les différents éléments (phonologique, morphologique, syntaxique et lexicale, et, en plus, un ensemble de normes socio-pragmatiques) qui sont nécessaires pour fonctionner en tant qu'outil pour la communication. C'est pour cette raison que l'expression "langue locale" est préférable à celle de dialecte.

Il faut également considérer qu'aucune de ces "langues locales" n'a jamais dépassé le niveau de l'usage exclusivement oral. Pour mieux dire, il peut arriver que, çà et là et à certains moments, certains locuteurs se soient exprimés dans l'écriture de leur langue locale: ce faisant cependant ils ne se sont jamais placés à l'intérieur d'une "tradition textuelle" qui ne fût celle de l'autre ou des autres langues de référence dont, conformément aux conditions de diglossie citées ci-dessus, ils sont également des utilisateurs, notamment pour l'écriture.

Une recommandation: notre besoin de regrouper et de classifier les langues ne devra pas être confondu par le lecteur avec une classification "naturelle". En d'autres mots, si nous serons forcés, pour les besoins de classement, de parler, par exemple, de "francoprovençal" (ou de "groupe francoprovençal"), ou de "walser" ou de "provençal alpin", le lecteur devra toujours considérer que l'on ne veut pas, par ces étiquettes, faire allusion à des langues vraies et propres, réelles et concrètes, mais tout simplement à des regroupements de facilité, qui assemblent d'une façon parfois même arbitraire des réalités linguistiques différentes, ayant toutefois certains traits communs.

Nous donnons ici encore deux avertissements, de caractère terminologique: lorsque nous parlons de «koïnè», nous nous référons, bien sûr, à une «langue commune», mais celle-ci peut être le résultat de la suprématie de la langue d'une localité, le résultat d'une sorte de «collage» entre plusieurs variétés, ou encore celui d'un échafaudage intellectuel d'un système abstrait. La deuxième précaution concerne la notion de «langue toit», par laquelle on entend un système linguistique «standardisé» qui domine, en tant que langue de la culture et modèle normatif, une ou plusieurs variétés dont il est un parent plus ou moins proche.

Dans le secteur occidental des Alpes, nous pouvons immédiatement observer en acte l'une des plus importantes et fréquentes "règles" de la situation linguistique alpine: la ligne de partage des eaux, qui à partir de la formation des grands "États-nations" a été employée pour tracer les soi-disant frontières naturelles (c'est la même ligne qui avec quelques exceptions marque aujourd'hui les limites entre la France et l'Italie à l'Ouest; puis, au Nord, entre la Suisse et l'Italie et entre l'Autriche et l'Italie et enfin, à l'Est, entre la Slovénie et l'Italie) ne joue pas le même 
Langues et dialectes dans l'arc alpin

T. Telmon, J. Médélice

LA FAMILLE LINGUISTIQUE ROMANE

\section{LE PROVENÇAL}

rôle pour ce qui concerne la détermination des frontières linguistiques. Le parler populaire de Barcellonette, sur le versant occidental (français) de la crête de faite, tout en constituant une langue locale à part entière, est pourtant plus apparenté au parler populaire de Demonte, à l'Est du Col de Larche, qu'au parler d'autres localités, même plus proches ou placées du même côté de la chaîne alpine.

La Fig. $n^{\circ} 1$ nous montre que trois grandes familles linguistiques (romane, germanique, slave) se partagent l'espace alpin: à l'intérieur de chacune d'elles, les linguistes distinguent d'habitude des groupes plus petits.

À l'exclusion du français, dont nous traiterons plus loin et qui, tout en étant lui-même, dans la France du nord, une continuation du latin, ne s'est affirmé qu'au Moyen Âge dans le milieu alpin, il s'agit toujours des continuations locales "naturelles" du latin vulgaire, modelé, selon des quantités et des modalités différentes, par des éléments de substrat, d'adstrat ou de superstrat. À partir des Alpes Maritimes, le premier groupe qu'on trouve est celui des parlers occitans.

Le regroupement des parlers occitans est très étendu et subdivisé en plusieurs sous-groupes. Dans l'aire alpine, c'est le provençal (et plus précisément le provençal alpin) le sous-groupe à qui reviennent les parlers des Alpes Maritimes et des Alpes Cottiennes, à partir du Col de Tende jusqu'au Col de Mongenève. Ce groupe occupe, dans le territoire français, toutes les vallées tributaires des bassins du Var et de la Durance; en territoire italien, les vallées de la province de Coni (Pesio et Ellero, Vermenagna, Gesso, Stura de Demonte, Grana, Maira, Varaita, $\mathrm{Po}$ ) et les plus méridionales de la province de Turin (Pellice, Cluson, Germanasca, Haute Vallée de Suse). Il faut remarquer l'absence totale de toute forme de koïnè ou de standardisation et l'inexistence de toute possibilité de reférence à une "langue-toit", soit du côté oriental soit de celui occidental. Pour donner une idée des aspects que la variabilité géographique du provençal alpin peut revêtir (mais le phénomène regarde également le francoprovençal, l'alémanique, le bavaro-autrichien, le ladin, le frioulan ou le slovène), on donne ici un exemple tout-à-fait casuel de la fragmentation lexicale. Les «épluchures» de la pomme de terre, peuvent se dire lo paraules, le paralhe, la paralhes, avec des diversités phonétiques et morphologiques mais avec le même type lexical, à Le Pin, Galaure, La Grave; elles deviennent cependant louz eploeshe à Crolles; mais aussi les pelures, lis espelouiro, las peluires à Charnècles, Saoû, Baratier; le peliotes à La Chapelle-de-Surieu et à St-Jean-de-Bournay; le pelalhes, la pelali, les pelayes à Allevard, La Mure, Aiguilles; lou trioun à Saoû; et encore: les pieräires à St-Véran; les plumàlias et les plumalhes à Gap, dans le Champsaur et à Laragne (2). 
Langues et dialectes

dans l'arc alpin

T. Telmon, J. MédéLice

\section{LE \\ FRANCOPROVENÇAL}

LE FRANÇAIS
En France, l'aire de cette famille linguistique, découverte et définie par le linguiste italien Graziadio Isaia Ascoli dans la deuxième moitié du XIXème siècle, occupe un espace qui s'étend vers l'Ouest au-delà de l'espace exclusivement alpin, jusqu'au Lyonnais et au Forez, dans la zone du Massif Central; en territoir alpin, les parlers que l'on peut attribuer à la famille francoprovençale vont du Grésivaudan (Daufiné septentrionale) à la Savoie, à la Suisse Romande et, à l'Est de la ligne de partage des eaux, des vallées de la province de Turin (Sangone, Basse Vallée de Suse, Cenischia, Stura de Viù et de Ala, Grande de Lanzo, Locana, Soana) jusqu'à tout le bassin valdôtain de la Doire Baltée (à l'exclusion de la vallée du Lys, dont le parler est walser (v. infra, au point 1.2). Les conditions de survie des parlers francoprovençaux sont assez différentes, selon les aires: réduites à quelques valétudinaires dans le territoire français, elles sont un peu plus vivantes en Suisse; sur le versant italien, elles sont encore assez vives en province de Turin et décidément vitales en Vallée d'Aoste. Tout comme dans les autres terroirs francoprovençaux, dans le Val d'Aoste n'a d'ailleurs jamais existé une tradition écrite, ni une tendance vers des formes d'unification: là aussi, la langue a toujours eu un usage exclusivement oral.

Contrairement aux autres deux composantes du groupe gallo-roman (occitan et francoprovençal), la présence desquels est sur les Alpes la continuation directe et ininterrompue du latin vulgaire, le français s'est ajouté comme un code ultérieur d'apprentissage; initialement, avec des fonctions

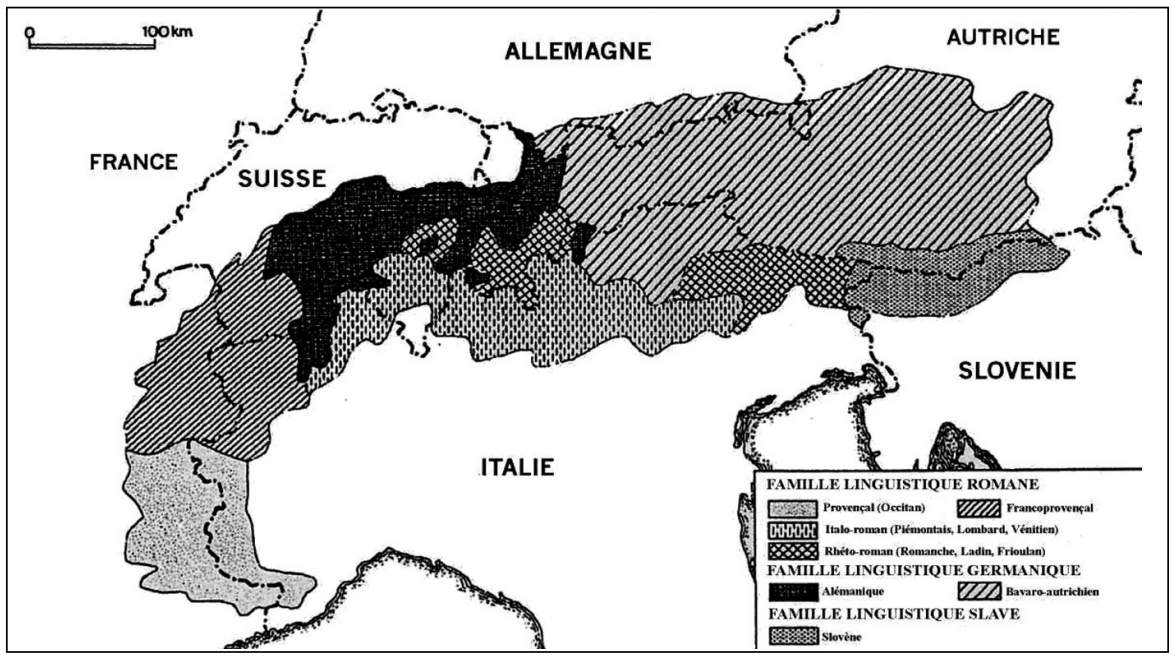


Langues et dialectes

dans l'arc alpin

T. Telmon, J. Médélice

LE GALLO-ITALIQUE de langue écrite. La langue française s'est de plus en plus répandue, à partir surtout de l'Ordonnance de Villers-Cotteret, par laquelle en 1539 le roi de France François Premier imposait l'emploi du français à la place du latin ou des langues vulgaires pour rédiger tout document officiel. Du côté oriental de la chaîne alpine, quelque chose de pareil fit aussi le duc Emanuel Filibert avec les "Lettere patenti" de 1561; avec cette différence que le duc de Savoie, conscient de gouverner un État inter-alpin et plurilingue, imposa l'emploi de «la lingua volgare» (c'est à dire du toscan, avec beaucoup de traits régionaux) dans ses Etats en deçà des Alpes et du français dans ses États transalpins. Alors que le français s'accorda assez tôt , sous la forme d'un bilinguisme presque parfait, avec les parlers provençaux du peuple dauphinois ou francoprovençaux des savoyards, litalien n'eut pas le même sort près des sujets du versant "italien". À quelques exceptions près, les montagnards des vallées provençales alpines et francoprovençales du Piémont continuèrent à ignorer l'italien jusqu'à très récemment. Les exceptions sont la Vallée d'Aoste, la Haute Vallée de Suse, le Haut Val Cluson, la Haute Vallée Varaita et les vallées dites "vaudoises". Pour des raisons différentes et souvent superposées l'appartenance d'abord au Dauphiné et puis à la France dans les cas des Vallées Varaita, Cluson et Suse; l'inclusion des valdôtains parmi les sujets francophones; le lien des vaudois avec le mouvement réformé dont le centre principale était la Genève francophone de Calvin), ce ne fut pas litalien la langue de leur bilinguisme, mais plutôt le français, qui eut ainsi la chance de devenir la langue du progrès social des classes populaires, de sorte que dans toutes ces vallées put se développer une vraie et propre alternative sociale dans le choix entre le code langue locale et le code langue française en tant que langue maternelle. C'était inévitable que, dans de telles conditions, le français commence à revêtir, un peu à la fois, des connotations particulières, conservant, d'un côté, de traits que le français "de France" a entre-temps abandonné et introduisant, de l'autre côté, des localismes et des emprunts de litalien ou des dialectes di Piémont. Parmi les exemples, on peut citer la tendance à l'articulation d'une voyelle muette en fin de mot, alors que la prononciation «correcte» la ferait disparaitre: [lynë] à la place de [lyn] («lune», la plupart des patois ayant huno, huna), [e'kolë] pour [e'kol] («école»; patois écòlo, eicòla), etc. Ou encore, l'articulation de la latérale palatale dans des mots où la palatalisation «parisienne» arrive à effa-

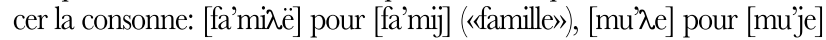
(«mouillen»), etc. Et pour le lexique: boureiller, bourreher «sellien»; bouter «poussen»; commerçable «vendable», etc.

Dans l'ensemble des dialectes de l'Italie du Nord, s'appellent gallo-italiques les dialectes du Piémont, de la Ligurie, de la Lombardie et de l'Émilie-Romagne. Tout en étant le résultat du latin appris par des populations d'origine celtique - 
Langues et dialectes

dans l'arc alpin

T. Telmon, J. Médélice

\section{LES PARLERS "PÉDÉMONTANS" DU PIÉMONT}

\section{LES DIALECTES DE LA LOMBARDIE}

\section{L'TTALO-ROMAN}

LES DIALECTES DE LA VÉNÉTIE pas moins que le gallo-roman qui comprend l'occitan, le francoprovençal et la langue "d'oil" - l'histoire linguistique, culturelle, administrative, économique de ces régions s'est orientée depuis toujours vers les grands centres de la culture et du pouvoir politique italiens. Par conséquent, leur évolution linguistique s'est développée différemment par rapport à celle de la famille gallo-romane. Les régions alpines de parler gallo-italique sont le Piémont et la Lombardie.

À vrai dire, à la partie alpine du Piémont appartient surtout son secteur occidental, c'est-à-dire celui dont les populations ont, come on vient de le voir, des parlers de type gallo-roman, que ce soit provençal alpin ou francoprovençal ou français. Pour ce qui regarde la partie restante de la région, les parlers de type alpin se bornent au Piémont septentrional, où les Alpes ont déjà la dénomination d'Alpes Centrales, et plus précisément d'Alpes Pennines. Ces parlers sont gallo-italiques. À leur intérieur, nous trouvons la variété des dialectes "valsesiani", dont les caractéristiques sont sensiblement éloignées de celles du turinois, variété de référence principale du type galloitalique "pédémontan», présentant certains traits qui les approchent aux dialectes de la Lombardie. Comme l'on verra plus loin (cfr. infra, prf. 1.2), dans ce même secteur du Piémont septentrional se trouvent aussi quelques colonies méridionales du type germanique alémanique.

Toutes les Préalpes Lombardes et le versant sud des Alpes Lepontines, comprenant le Canton suisse du Tessin et les provinces de Varese, Como et Sondrio en Lombardie sont habités par des populations dont les parlers lombards ne sont pas moins différenciés, à leur intérieur, des parlers «pédémontans» du Piémont. On peut en effet y relever au moins trois variétés différentes: le "brianzolo" dans la zone des Préalpes et des grands lacs de Como et de Lugano; le tessinois, à son tour très fragmenté mais en même temps assez proche du «brianzolo»; le «valtellinese», beaucoup plus éloigné que les autres du modèle urbain de Milan.

Dans les régions italiennes Trentino-Alto Adige, Veneto et Friuli Venezia Giulia, les parlers qui n'appartiennent pas aux regroupements ladins et friulans ou aux communautés germaniques ou slaves (dont on parlera plus loin), sont du type italo-roman. Le substrat de ces parlers n'est pas celtique, comme dans les parlers du Piémont et de la Lombardie, mais vénitien, et les issues historiques des développements linguistiques ont abouti à des parlers beaucoup moins éloignés des parlers toscans que les parlers «pédémontans» et lombards.

Les dialectes de la Vénétie sont eux aussi très différenciés. Ils s'étendent sur les Préalpes de la Vénétie (Monti Lessini, Haut plateau d'Asiago, Monte Grappa, Préalpes Bellunesi, Préalpes 
Langues et dialectes

dans l'arc alpin

T. Telmon, J. Médélice

\section{LES DIALECTES DE LA PROVINCE DE TRENTE}

\section{LE RHÉTO-ROMAN}

\section{LE ROMANCHE}

\section{LE LADIN}

Juliennes), sur le Carso (entre la basse vallée de l'Isonzo et le Quarnaro): les variétés linguistiques les plus remarquables sont celles du Haut Vicentino, du Bellunese, du Triestino.

Les dialectes italo-romans de la province de Trente, repartis sur les Alpes et les Préalpes à partir du Passo del Tonale et des Giudicarie jusqu'à la moyenne et basse vallée de l'Adige, sont des variétés de transition entre les dialectes lombards orientaux (Haut Bergamasco, Bresciano) et les dialectes vénitiens qu'on vient de décrire.

Pour plus de commodité de classement, nous suivons ici la tradition scientifique allemande, qui classe en une même famille tous les parlers de l'Engadine et des Grisons suisses, ceux du groupe étalé autour du massif montagneux du Sella et ceux du Frioul: trois groupes qui n'ont jamais connu aucune unité, ni politique, ni culturelle, ni géographique.

C'est le plus occidental des trois groupes linguistiques rhéto-romans. Il occupe le territoire du Canton des Grisons, situé à nord-nord-ouest du Tessin, dans les hautes bassins du Rin et de l'Inn, au cœur des Alpes Centrales, et plus précisément des Rhétiques. Dans le système linguistique de la Suisse, le romanche jouit du statut de "langue officielle" de la Confédération, quoiqu'il n'existe (ou, pour mieux dire, n'ait existé jusqu'à il y a quelques années) aucune forme de koiné, et bien que l'on puisse déterminer cinq grandes variété: sursibuan, sutsilban, surmiran, haut engadinois et bas engadinois, à leur tour fragmentées en des parlers locaux. Pour arrêter le déclin de cette famille linguistique et son recul face à la propagation de l'allemand, les autorités fédérales et cantonales ont déployé de grands efforts; malgré cela, même dans la petite capitale Coire (lat. Curia Raetorum; en romanche Cuera et en allemand Chur) de nos jours est beaucoup plus utilisé l'allemand que le romanche: que ce soit le Rumantsch grischun souhaité comme köné par la Lia Rumantscha ("Ligue romanche"), ou celui de la variété locale.

Strictement parlant, l'emploi de ce glossonyme devrait se limiter à la seule localité de San Martino in Badia, où ladin est effectivement la dénomination populaire de la langue locale. Selon la coutume des linguistes, cette dénomination a été élargie jusqu'à comprendre tous ces parlers (ayants pourtant des caractères considérablement différentes par rapport aux dialectes italo-romans de la province de Trente et de la Vénétie septentrionale) qui se sont développés du latin dans le système de vallées qui pivote autour du massif du Sella, dans les Dolomites. Les vallées où les langues ladines sont aujourd'hui vivantes - elles aussi à l'état d'oralité presque exclusive, malgré de récentes démarches d'emploi littéraire et d'unification orthographique - sont les vallées Gardena et 
Langues et dialectes

dans l'arc alpin

T. Telmon, J. Médélice

\section{LE FRIOULAN}

\section{LA FAMILLE LINGUISTIQUE GERMANIQUE}

\section{L'ALÉMANIQUE}

LE BAVAROAUTRICHIEN
Badia, en province de Bolzano; Fassa (province de Trente), Cordevole (province de Belluno) et, selon une conception élargie, aussi Boite et haut Piave (province de Belluno).

C'est la "section orientale" du groupe rhéto-roman. De même que les variétés ladines, les variétés alpines du frioulan sont au sud de la ligne de partage des eaux; les plus remarquables sont les variétés de la Carnia, sur les Alpes Carniques, entre le Passo di Monte Croce di Comelico et le Passo di Camporosso. Des parlers de type frioulan sont cependant présents aussi sur les Alpes Juliennes septentrionales, parfois en alternance avec des parlers de la famille slave. Des trois regroupements rhéto-romans, le frioulan est probablement le plus homogène et compact, tant et si bien qu'il a été aussi celui qui s'est le plus approché de la formation d'une koïné. À l'élargissement de cette koöné à la région tout entière se sont opposées d'abord les variétés marginales, hostiles à accepter la proposition d'un frioulan unitaire sur la base de la variété centrale, et surtout la carence de lexiques techniques spécifiques, autres que celui des activités agricoles.

Comme l'on peut observer dans la Fig. 1, la famille linguistique germanique est, sur les Alpes, la plus nombreuse et répandue. Abstraction faite de quelques exceptions, dont on traitera plus loin, elle est entièrement placée au nord de la ligne de partage des eaux, sur les versants suisse, allemand et autrichien de la chaîne alpine, des Alpes Centrales aux Orientales. Les regroupements qui en font partie sont trois, tous appartenant au "haut-allemand": alémanique, bavaro-autrichien et carinthien.

En partant du côté oriental du Canton suisse du Valais (le côté occidental est romand, de parler francoprovençal et/ou d'oil), l'ensemble des parlers germaniques de souche alémanique s'étend sur tout le versant nord des Alpes Centrales, jusqu'au Lichtenstein et aux Alpes de l'Autriche occidentale. Une partie des populations de parler alémanique s'identifie avec la dénomination de walser (contraction de walliser "valais"). Au sud de la crête alpine, des colonies alémaniques, qui se sont fixées dans la vallée valdôtaine du Lys (Gressoney St Jean, Gressoney La Trinité, Issime), en Valsesia (Alagna, Rima, Rimella), dans l'Ossola (Macugnaga, Ornavasso - désormais assimilé aux parlers romans environnants - et Formazza) et dans le village tessinois de Bosco Gurin, prennent aussi bien la dénomination de walser.

C'est le groupe germanique le plus nombreux. Il s'étale sur une partie des Alpes Rhétiques, sur les Alpes et les Préalpes Bavaroises, sur les Alpes Nordiques et sur les Préalpes du Salzburgeois, de l'Autriche et de la Styrie. De nombreux groupes 
Langues et dialectes

dans l'arc alpin

T. Telmon, J. Médélice

\section{LE CARINTHIEN}

\section{LA FAMILLE LINGUISTIQUE SLAVE}

\section{LE SLOVÈNE}

bavaro-autrichiens se trouvent aussi au sud des Alpes: le plus important est celui qui en plusieurs vagues s'est installé, à partir de l'époque médiévale, dans le territoire qui s'appelle aujourd'hui Tyrol méridional ou Haut Adige (correspondant à la province de Bozen/Bolzano, à l'exception près des vallées ladines de Gardena e Badia). Hors de la province de Bolzano, le long d'un couloir qui de la vallée du Fersina se prolonge jusqu'aux collines du Veronais, nous trouvons encore quatre noyaux de populations de parler bavaro-autrichienne. Le premier est celui des "mòcheni", fixés en Val Fersina, dans le Haut Perginese (communes de Frassilongo, Roveda, Fierozzo, Palù); le deuxième celui de Luserna (dans le dialecte local Luzern), aujourd'hui isolé, le troisième noyau est celui du plateau d'Asiago, siège de la "Ligue des sept communes vicentins" (réduits aujourd'hui à Roana, Rotzo et Gallio); le dernier est sur les collines veronaises, siège de la «Ligue des Treize Communes véronais», l'autonomie desquels fut longtemps reconnue par la République de Vénise (réduits désormais à la seule commune de Selva di Progno, ou mieux au seul hameau de Giazza). Sensiblement différente est par contre l'origine de a colonie de Sappada (Plodn), à propos de laquelle il parait qu'il faut parler d'une émigration "par ricochet", de colons provenant du Val Pusteria.

Entre les Dolomites, les Alpes Carniques, le Carso, les Noriques et les Caravanches se place infin le groupe germanique carinthien. Le noyau plus nombreux de ce groupe occupe le haut bassin de la Drava, mais, tout comme pour le bavaro-autrichien, quelques colonies se trouvent aussi sur le versant italien de la chaîne alpine. Il s'agit d'établissements de paysans, bûcherons, mineurs, datables autour du XIII-XIV siècle, localisés dans le Frioul à Sauris (dans le dialecte local Zahren), à Timau, hameau de la commune de Paluzza, et dans le Val Canale, de Pontebba à Tarvisio.

Elle s'étale des Alpes Juliennes à l'extrémité sud-orientale de la chaîne alpine, avec les Caravanches, les Alpes de Kamnik et le Pohorje. Du point de vue politique, la plupart du groupe slave est concentré dans la République Slovène, mais il forme une minorité linguistique aussi en Autriche (Styirie et Carinthie) et en Italie.

Dans la famille des langues slaves, c'est le groupe slovène que nous trouvons tant sur les Alpes de la Slovénie, que sur le Alpes autrichiennes et italiennes. Pas moins que les autres groupes qu'on a vus jusqu'ici, ce groupe aussi est subdivisé en de nombreuses variétés locales, dont la physionomie est parfois assez éloignée de celle du slovène standard, notamment dans les zone alpines plus hautes et isolées, ou dans les aires où le slovène se trouve dans des conditions sociolinguistiques subalternes par rapport à des langues d'état différentes (l'allemand en Autriche, l'italien au Frioul oriental). Dans cette dernière région, les parlers slovènes sont concentrés surtout dans les terroirs de frontière avec la Slovénie, où 
Langues et dialectes

dans l'arc alpin

T. Telmon, J. Médélice

\section{LES LANGUES D'EMPLOI OFFICIEL}

\section{LA FAMILLE LINGUISTIQUE ROMANE}

\section{LE FRANÇAIS}

LITALIEN ils forment ce qu'on appelle «Beneska Slovenija» ou «Slavia Veneta» («Slavie vénitienne»), qui correspond à la Vallée du Resia, aux hautes vallées du Torre et du Natisone et au haut Isonzo.

La caractéristique des langues populaires est donc celle d'une tendance marquée à la dialectalité. Cette dialectalité se révèle à travers: a) un emploi presque exclusivement parlé; b) une tendance à l'identification entre la langue et le village; $c$ ) la fragmentation; d) l'absence ou la marginalisation de koïnés ou de tendances à la standardisation. Les langues officielles, c'est-à dire les langues qui l'emportent dans les usages des administrations des États à qui appartiennent les territoires alpins qu'on a vus jusqu'ici, sont au contraire assez vivement standardisées et visent à revêtir, dans la hiérarchie des répertoires linguistiques, les positions les plus hautes, occupées par les emplois de type formel. A quelques exceptions près, nous trouverons donc des "langues-toit" d'État appartenant à la famille romane où les langues populaires sont elles-mêmes de type roman; par contre, nous trouverons l'allemand où les langues populaires sont de type germanique et le slovène où les parlers locaux sont eux-mêmes slaves.

Si nous revenons dans le secteur occidental, nous pouvons observer, à un tout premier coup, que la ligne de partage des eaux qui était normalement enjambée par les parlers provençaux alpins et francoprovençaux, redevient une frontière plutôt nette. Sur le versant français des Alpes Maritimes, Cottiennes et Graiennes, en effet, c'est uniformément le français qui est la langue officielle de l'État; à l'est, c'est l'italien, à l'exception de la Vallée d'Aoste, où, grâce au Statut spécial et à l'autonomie régionale qui en découle, le français et l'italien sont toutes deux considérées langues officielles. Après la promulgation, de la part du Parlement italien, de la loi 482 de 1999 pour la protection des "minoranze linguistiche storiche", font exception aussi ces communes des Vallées vaudoises, du Val Cluson et de la Vallée de Suse qui ont décidé leur appartenance à une minorité linguistique de langue soit française soit occitane (ou francoprovençale). Également française est la langue officielle de ces cantons suisses (Suisse Romande) dont les langues locales sont, par contre, francoprovençales.

L'italien est, comme on vient de le dire, langue officielle dans l'aire alpine du Piémont, où les langues populaires appartiennent pourtant aux groupes occitan, francoprovençal ou français. À côté du français, il est la langue officielle du Val d'Aoste. Dans les Alpes Centrales, c'est la langue officielle dans toutes les aires où des dialectes de type gallo-italique sont parlés populairement (y compris le Canton suisse du Tessin). Dans le 
Langues et dialectes

dans l'arc alpin

T. Telmon, J. Médélice

\section{LE ROMANGHE}

\section{LA FAMILLE LINGUISTIQUE GERMANIQUE \\ L'ALLEMAND (HOCHDEUTCH)}

LA FAMILLE LINGUISTIQUE SLAVE

LE SLOVÈNE

L'OFFICIALISATION DES LANGUES MINORITAIRES HISTORIQUES EN ITALIE
Haut Adige/Sud Tyrol est prévu un bilinguisme officiel, comprenant l'allemand (Hochdeutsch) et l'italien. Dans le secteur oriental des Alpes, l'italien est la langue officielle au Frioul, même là où les dialectes frioulans, carinthiens ou slovènes constituent le patrimoine linguistique populaire.

On avait déjà remarqué (cfr. supra, prf. 1.1.6.1) que le Canton des Grisons est marqué par l'existence, au niveau de langues parlées locales, d'un groupe linguistique roman particulier, dit "rhéto-roman". Quoique les parlers attribuables à ce groupe soient de plus en plus perdants, face à la diffusion de l'allemand, et bien que les efforts pour constituer et imposer une variété de koïné ne se soient pas révélés très efficaces, la Confédération suisse a reconnu aussi le Romanche comme langue officielle, à côté du français, de l'allemand et de l'italien.

Dans les Alpes Centrales, l'allemand littéraire est la langue officielle de la Suisse allemande. Dans le secteur oriental des Alpes, l'allemand est également la langue officielle dans le Liechtenstein, en Bavière, en Autriche et, en alternance bilingue avec l'italien, dans la province de Bolzano/Bozen. Pour ce qui regarde, en particulier, la Suisse allemande, il faut cependant observer que le Schwitsertiutsch, variante régionale d'allemand dont la base est constituée par les parlers populaires hautallemands de type alémanique, est de plus en plus répandue et standardisée, même aux niveaux de l'officialité et de la formalité.

Sur les Alpes, le slovène est la langue officielle de la République slovène, une bonne partie de laquelle appartient à l'aire alpine des deux chaînes qui vont des Alpes Juliennes aux Dinarides et, plus au nord, des Caravanches aux Alpes de Kamnik et au Pohorje.

Depuis le 15 décembre 1999, date de l'approbation de la loi de l'État italien pour la sauvegarde des minorités linguistiques historiques, dans les communes qui ont décidé leur appartenance à une des minorité prévue par l'article 2 de la loi («les populations albanaises, catalanes, germaniques, grecques, slovènes et croates, et celles parlant le français, le francoprovençal, le frioulan, le ladin, l'occitan et le sarde») toutes les langues populaires dont on a parlé au premier paragraphe (sauf les gallo-italiques et les italo-romans) ont assumé, en toute rigueur, une forme d'officialité, puisqu'elles peuvent être utilisées, par exemple, dans les conseils et dans les bureaux municipaux. Il reste à résoudre le nœud de la/des variété/variétés: si le but de la loi est celui de sauvegarder des minorités, l'hypothèse de privilégier une variété au détriment des autres, ou de 
Langues et dialectes dans I'arc alpin

T. TeLmon, J. MédéLICE créer artificiellement une autre variété «de référence» ou bien encore, de chercher à l'extérieur du terroir une variété douée d'un prestige particulier, semble alors très peu plausible et surtout très peu cohérente avec ses fins.

\section{NOTES}

(1) Cet article est d'abord paru en 2006 dans la partie «Les langues des Alpes», in C. Jourdain Annequin (a c. di). Aires culturelles, aires linguistiques dans les Alpes occidentales. Actes du Colloque de Grenoble, 18-19 novembre 2004. p. 127-140, Grenoble: Glénat.

(2) Pour une lecture correcte des mots dialectaux exposés ci-dessus, il faut remarquer que, étant donné que la plupart d'eux porte l'accent de mot sur la pénultième syllabe (non pas sur la dernière, comme c'est la règle pour la langue française), les voyelles ou les diphtongues soulignées sont par conséquent les porteuses de l'accent du mot. En plus, le lecteur français doit toujours prononcer les «-s du pluriel» en fin de mot, et articuler comme voyelles «pleines» les «-e-») qui les précèdent. 